Well adapted normal linearization in singular perturbation problems

Non Peer-reviewed author version

BONCKAERT, Patrick; DE MAESSCHALCK, Peter \& DUMORTIER, Freddy (2011)

Well adapted normal linearization in singular perturbation problems. In: Journal of Dynamics and Differential Equations, 23(1). p. 115-139.

DOI: $10.1007 / \mathrm{s} 10884-010-9191-0$

Handle: http://hdl.handle.net/1942/11635 


\title{
Well adapted normal linearization in singular perturbation problems
}

\author{
P. Bonckaert P. De Maesschalck \\ F. Dumortier \\ Hasselt University, Campus Diepenbeek \\ Agoralaan, gebouw D \\ B-3590 Diepenbeek, Belgium
}

October 1, 2010

\begin{abstract}
We provide smooth local normal forms near singularities that appear in planar singular perturbation problems after application of the well-known family blow up technique. The local normal forms preserve the structure that is provided by the blow-up transformation. In a similar context, $C^{k}$ structure-preserving normal forms were shown to exist, for any finite $k$. In this paper, we improve the smoothness by showing the existence of a $C^{\infty}$ normalizing transformation, or in other cases by showing the existence of a single normalizing transformation that is $C^{k}$ for each $k$, provided one restricts the singular parameter $\varepsilon$ to a ( $k$-dependent) sufficiently small neighborhood of the origin.
\end{abstract}

2000 Mathematics Subject Classification: 34C20, 34C14.

Keywords: smooth normal linearization, vector field, line of singularities, singular perturbations

Dedicated to the memory of Jack Hale

\section{Introduction}

This paper deals with normal forms for families of vector fields that often arise in the study of planar singular perturbation problems. Though the paper has a non-void intersection with [DR], it is largely complementary and independent. Also the main idea of the proof is quite different. The vector fields under study typically have a singular point with one nonzero eigenvalue of the linear part and multiple center and/or parameter directions. Either such singular points appear naturally in the singular perturbation problem (normal hyperbolicity) under consideration, or they arise from studying a more complicated singular point without any nonzero eigenvalue in the linear part (see e.g. [DMD10] or [DMD11] for motivating examples). With the blow-up technique introduced in [DR96] one often succeeds in reducing the study of such a complicated singular point to the study of one or more partially hyperbolic points. In this blowup process, it is necessary to include parameters in the phase-space (thereby 
explaining the more-dimensional center directions in planar problems), and to replace these parameters by a product of parameters and phase variables. A blow-up transformation might for example by given by $(x, y, \lambda):=(X, X Y, X Z)$. The trivial foliation $d \lambda=0$ is then replaced by a more complicated foliation $d(X Z)=0$. This complicates the dynamics in the center directions, but the property of having one hyperbolic direction is an important advantage, see also Remark 2 below. The objective in this paper is to normally linearize the vector field, i.e. to linearize the differential equation of the hyperbolic variable, leaving unchanged every fiber of the stable (resp. unstable) foliation, as well as the extra structure given by the foliations mentioned above. While a finitely smooth normal linearization of the systems has already been dealt with in a general setting (see e.g. [Tak71], [Bon96], [Bon97]), we aim in this paper at getting a normal linearization that is as smooth as possible. The theorems that we present, especially Theorem 2, provide normal linearizations that respect a lot of essential structure, combined with a maximal smoothness. With these theorems, some proofs of former results can be made more direct and transparent. Moreover, Theorem 2 reveals to be essential for proving the new results that can be found in [DMD10], [DMD11], and some work in progress. We will deal with specific situations that we describe now.

One result that is presented in this paper deals with the $(\varepsilon, \lambda)$-family of vector fields

$$
X_{\varepsilon, \lambda}:\left\{\begin{array}{rl}
\dot{x} & =-x \varepsilon^{r} m\left(x, y_{1}, \ldots, y_{n}\right) \\
\dot{y_{i}} & =q_{i} y_{i} \varepsilon^{r} m\left(x, y_{1}, \ldots, y_{n}\right), \\
\dot{z} & =-b(\lambda) z+f\left(x, y_{1}, \ldots, y_{n}, z, \varepsilon, \lambda\right) .
\end{array} \quad i=1, \ldots, n,\right.
$$

where $q_{i}>0, r \geq 1$, and $m$ is a monomial in $x, y$ of the form $\pm x^{\kappa_{0}} y_{1}^{\kappa_{1}} \ldots y_{n}^{\kappa_{n}}$ (with $\kappa_{i} \geq 0$ ); in case all $\kappa_{i}=0$ we suppose $m= \pm 1$. (At the end of this section, in Remark 3, we comment on the restriction on $m(x, y)$.) The function $f$ is $C^{\infty}$ near $x=y=z=\varepsilon=0$ and for $\lambda \in \Lambda$ where $\Lambda$ is a compact subset of some finite-dimensional euclidean space. We keep $\varepsilon \geq 0$. The function $b(\lambda)$ is $C^{\infty}$ on $\Lambda$. Furthermore we assume that $b(\lambda)>0$, for all $\lambda \in \Lambda$, and that

$$
f(0,0,0,0, \lambda)=0, \quad \frac{\partial f}{\partial z}(0,0,0,0, \lambda)=0
$$

A first result concerns the existence of "center manifolds" at the origin with nice differentiability properties. We mean $(\varepsilon, \lambda)$-families of invariant manifolds $z=\varphi(x, y, \varepsilon, \lambda)$ that form $\lambda$-families of center manifolds of the extended family of vector fields

$$
X_{\varepsilon, \lambda}+0 \frac{\partial}{\partial \varepsilon}
$$

at $(x, y, \varepsilon, \lambda)=(0,0,0,0)$. Throughout the paper, we will abusively call such invariant manifolds center manifolds of $X_{\varepsilon, \lambda}$ at the origin, though in case $m \equiv$ \pm 1 they are not center manifolds, but merely invariant manifolds.

Of course, for each $k \geq 1$, the existence of $C^{k}$ local center manifolds of (2) is well-known, and it is known that in general one cannot expect the existence of a $C^{\infty}$ center manifold, even if one starts with an analytic vector field (see [vS79]). In fact, another counterexample is given in [Tak86]; there the author provides an example of a smooth vector field in which any given center manifold cannot be further smoothened by restricting it to a smaller domain. Nevertheless, in 
the specific family expressed by (1), we can improve the result of the center manifold theorem: we obtain the existence of a center manifold given as the graph of a function that is $C^{1}$ on a large domain around the origin, and that for each $k$ is $C^{k}$ on a sufficiently small neighborhood of the chosen singular point. Moreover, in this specific problem, the neighborhoods, depending on $k$, will only shrink in the $\varepsilon$-direction when the smoothness requirements increase.

Theorem 1. Let $X_{\varepsilon, \lambda}$ be as in (1), with the conditions described above. Then there exists a neighborhood $V \times\left[0, \varepsilon_{1}\right]$ of $(x, y, \varepsilon)=(0,0,0)$ and a $C^{1}$-graph $z=\varphi(x, y, \varepsilon, \lambda)$ on $V \times\left[0, \varepsilon_{1}\right] \times \Lambda$ that forms an $(\varepsilon, \lambda)$-family of invariant manifolds of $X_{\varepsilon, \lambda}$, with $\varphi(0,0, \varepsilon, \lambda)=0$.

When $m \not \equiv \pm 1$, the function $\varphi$ can be chosen $C^{\infty}$.

When $m \equiv \pm 1$, there exists a decreasing sequence $\left(\varepsilon_{k}\right)_{k}$ for which $\varphi$ is $C^{k}$ on $V \times\left[0, \varepsilon_{k}\right] \times \Lambda$. Furthermore, in case $m \equiv 1, \varphi$ is smooth for $x \neq 0$ and in case $m \equiv-1, \varphi$ is smooth for $\|y\| \neq 0$.

Though the traditional center manifold theorem also shows the existence of $C^{k}$-center manifolds, for any desired value of $k$, the obtained center manifold is possibly dependent on $k$. This proves to be a nuisance in some applications. The main benefit of Theorem 1 is hence the fact that a single center manifold is constructed that can be made as smooth as required, provided one takes $\varepsilon$ small enough.

After straightening the center manifold given in Theorem 1, one gets a family of vector fields

$$
X_{\varepsilon, \lambda}:\left\{\begin{array}{rl}
\dot{x} & =-x \varepsilon^{r} m(x, y) \\
\dot{y}_{i} & =q_{i} y_{i} \varepsilon^{r} m(x, y) \\
\dot{z} & =-a(x, y, \varepsilon, \lambda) z+F(x, y, z, \varepsilon, \lambda) z^{2}
\end{array} \quad i=1, \ldots, n,\right.
$$

The functions $a$ and $F$ are $C^{k}$ for $(x, y) \in V,|z|$ small, $\varepsilon \in\left[0, \varepsilon_{k}\right], \lambda \in \Lambda$, and they are smooth for $x \neq 0$ in case $m \equiv 1$, for $\|y\| \neq 0$ in case $m \equiv-1$. Furthermore, $a(0,0,0, \lambda)=b(\lambda)>0$, and when $m \not \equiv \pm 1$, we may take $\varepsilon_{k}=\varepsilon_{1}$ (implying $C^{\infty}$ smoothness in a full neighborhood of the origin).

Theorem 2. Consider a family of vector fields (3), with the above mentioned conditions. There exists a local $C^{1}$-family of diffeomorphisms of the form

$$
(x, y, z) \rightarrow(x, y, \tilde{z}), \tilde{z}=\psi(x, y, z, \varepsilon, \lambda),
$$

with $\frac{\partial \psi}{\partial z}(x, y, 0, \varepsilon, \lambda)=1$, defined for $(x, y, z) \in \tilde{V}$ near the origin and for $\varepsilon \in$ $\left[0, \tilde{\varepsilon}_{1}\right], \lambda \in \Lambda$, conjugating the family (3) to

$$
\left\{\begin{aligned}
\dot{x} & =-x \varepsilon^{r} m(x, y) \\
\dot{y_{i}} & =q_{i} y_{i} \varepsilon^{r} m(x, y) \\
\dot{\tilde{z}} & =-a(x, y, \varepsilon, \lambda) \tilde{z}
\end{aligned}\right.
$$

$$
i=1, \ldots, n
$$

When $m \not \equiv \pm 1$ and (3) is $C^{\infty}$, then the conjugacy can be chosen $C^{\infty}$.

When $m \equiv \pm 1$, there exists a decreasing sequence $\left(\tilde{\varepsilon}_{k}\right)_{k}$ (with $\tilde{\varepsilon}_{k} \leq \varepsilon_{k}$ ) for which $\psi$ is $C^{k}$ on $\tilde{V} \times\left[0, \tilde{\varepsilon}_{k}\right] \times \Lambda$. The function $\psi$ is $C^{\infty}$ for $x .\|y\| \neq 0$.

Remark 1. In case $m \equiv 1$, one might expect the conjugacy to be smooth outside $x=0$ (and not just outside $x .\|y\|=0$ as announced in the theorem), in analogy 
with the smoothness of the center manifold in that case. This may be not true: lack of smoothness for nonzero values of $\varepsilon$ might also occur along $y=0$. Similarly, lack of smoothness for nonzero values of $\varepsilon$ might occur along both $x=0$ and $y=0$ in case $m \equiv-1$.

In the proof of this specific normal form, we use a more general statement on normal linearization of finite differentiability:

Theorem 3. For any $k$ there exists a $N=N(k)$ with the following property. Consider a $C^{N} \lambda$-family of vector fields on $\mathbf{R}^{q+1}$,

$$
X_{\lambda}(v, z)=F(v, \lambda) \frac{\partial}{\partial v}+G(v, z, \lambda) z \frac{\partial}{\partial z},
$$

defined for $v$ near 0 in $\mathbf{R}^{q}$, for $z$ near 0 in $\mathbf{R}$, and for $\lambda \in M$, where $M$ is a compact subset of a finitely dimensional euclidean space $\mathbf{R}^{p}$. Assume that $F(0, \lambda)=0$ and $\inf \{|G(0,0, \lambda)|: \lambda \in M\}>0$. There exists $\delta=\delta(k)>0$ such that if

$$
\sup \left\{|\operatorname{Re} \mu|: \mu \in \operatorname{Spec}\left(\frac{\partial F}{\partial v}(0, \lambda)\right) \text { and } \lambda \in M\right\} \leq \delta
$$

then there exist an open neighborhood $V_{k}$ of the origin in $\mathbf{R}^{q+1}$ and a local $C^{k+1}$ family of diffeomorphisms of the form

$$
(v, z) \rightarrow(v, \tilde{z}), \tilde{z}=z(1+\psi(v, z, \lambda)),
$$

with $\psi(v, 0, \lambda)=0$, conjugating the family $X_{\lambda}$ to the family

$$
N X_{\lambda}(v, \tilde{z})=F(v, \lambda) \frac{\partial}{\partial v}+G(v, 0, \lambda) \tilde{z} \frac{\partial}{\partial \tilde{z}} .
$$

Theorem 3 gives a way to normally linearize a vector field, just like in Theorem 2. However, as the smoothness of the normalizing transformation increases, the domain on which it is defined may shrink to a point in Theorem 3. Furthermore, the normalizing transformation itself might depend on the choice of $k$. Theorem 2 provides, in a more specific context, a single conjugacy with an increasing smoothness on decreasing domains, and furthermore, the decrease in domain only occurs in the $\varepsilon$-direction. The proof of Theorem 3 is inspired by techniques in [Bon97].

Remark 2. The fact that the hyperbolic direction $\frac{\partial}{\partial z}$ is one-dimensional implies that there are no resonances in this direction; this is used in the proof of Theorem 3 (e.g. estimate (20)). Our results might remain true if the $z$-direction is higher dimensional, provided that additional non-resonance assumptions on the real parts of the eigenvalues are made, see also [Bon97].

Remark 3. The class of vector fields (1) that Theorems 1 and 2 apply to is of a specific nature. Besides the assumption that the hyperbolic direction is one-dimensional, as explained in Remark 2, the family of vector fields (1) has some additional structure that limits the results to the specific form in (1). The presence of an invariant foliation $d\left(x^{q_{1}} y_{1}\right)=\cdots=d\left(x^{q_{n}} y_{n}\right)=0$ is a very important assumption; the necessity to have results on normal forms preserving this structure forms the basis of this paper. Such invariant foliations occur frequently in the study of degenerate singularities of families of vector fields. 
Another restriction on the generality of (1) is the nature of the monomial $m(x, y)$. Also this condition arises from the needs that become apparent when blowing up degenerate singularities in the context of singular perturbations. One could think of generalizing the results by replacing $m(x, y)$ by a more general function $m(x, y, z)$. It is however likely that the results in Theorem 1 and Theorem 2 are no longer valid (though we do not give an example) when $m$ depends on $z$. Also replacing $m(x, y)$ by a function that is zero along arbitrary curves through the origin will for sure cause complications, especially at points where the zero set $\{m=0\}$ is tangent to the invariant foliation.

\section{Proof of Theorem 3}

In the proof we will use cut off functions, among others, in the $(v, \lambda)$-direction (i.e. the "center direction"). It can be checked that we shall be able to choose those cut off functions not depending on the parameter $\lambda \in M$, due to the assumptions in the theorem. Hence, for the sake of readability, we will introduce a variable $x=(v, \lambda) \in \mathbf{R}^{m}:=\mathbf{R}^{q} \times \mathbf{R}^{p}$ that we will use when no confusion is possible. During the proof, we however take care that the constructed conjugacies are defined for $x=(v, \lambda) \in V \times M$, in some open neighborhood $V \subset \mathbf{R}^{m}$ of $v=0$. We thus write

$$
X(x, z)=F(x) \frac{\partial}{\partial x}+G(x, z) z \frac{\partial}{\partial z}
$$

and develop

$$
G(x, z)=G(x, 0)+G_{1}(x, z) \cdot z
$$

so (5) becomes

$$
X(x, z)=F(x) \frac{\partial}{\partial x}+\left(G(x, 0) \cdot z+G_{1}(x, z) \cdot z^{2}\right) \frac{\partial}{\partial z} .
$$

Let us abbreviate

$$
c:=\inf _{\lambda \in M}|G((0, \lambda), 0)|
$$

Suppose, by induction on $k$, that $X$ is of the form

$$
X(x, z)=F(x) \frac{\partial}{\partial x}+\left(G(x, 0) \cdot z+G_{1}(x, z) \cdot z^{k}\right) \frac{\partial}{\partial z} .
$$

We claim that we may, and will, assume that $G_{1}(x, 0)$ is 'as flat as needed' in the $v$-variable:

Lemma 1. Up to a polynomial change of variables we may assume that

$$
G_{1}(x, 0)=O\left(v^{\left[\frac{c}{\delta}\right]-1}\right), \quad \text { as } v \rightarrow 0 .
$$

Proof. Using condition (4) we infer that a monomial of the form $a(\lambda) v^{j_{1}} z^{j_{2}}$ is non-resonant whenever $1 \leq\left|j_{1}\right|<c / \delta$ and $j_{2} \geq 2$, because for the spectrum $\left\{\mu_{1}(\lambda), \ldots, \mu_{m}(\lambda)\right\}$ of $\frac{\partial F}{\partial v}(0, \lambda)$ we have

$$
\left|\left\langle\left(\operatorname{Re} \mu_{1}(\lambda), \ldots, \operatorname{Re} \mu_{m}(\lambda)\right), j_{1}\right\rangle\right| \leq \delta\left|j_{1}\right|<c \leq|G((0, \lambda), 0)| \cdot\left(j_{2}-1\right)
$$

and so

$$
G((0, \lambda), 0) \neq\left\langle\left(\mu_{1}(\lambda), \ldots, \mu_{m}(\lambda)\right), j_{1}\right\rangle+G((0, \lambda), 0) j_{2} .
$$

Hence the monomial $a(\lambda) v^{j_{1}} z^{j_{2}} \frac{\partial}{\partial z}$ is non-resonant. This proves the lemma. 
Let us write

$$
X^{0}(x, z)=F(x) \frac{\partial}{\partial x}+G(x, 0) \cdot z \frac{\partial}{\partial z}
$$

and

$$
G_{1}(x, z)=a(x)+O(z)
$$

and also

$$
X^{1}(x, z)=G_{1}(x, z) \cdot z^{k} \frac{\partial}{\partial z}=\left(a(x) \cdot z^{k}+O\left(z^{k+1}\right)\right) \frac{\partial}{\partial z}
$$

so $X=X^{0}+X^{1}$. Using cut off functions we may, and do, assume that all occurring flows are globally defined. We also assume, without loss of generality, that $G((0, \lambda), 0)$ is negative, i.e. $G((0, \lambda), 0) \leq-c$ with $c>0$.

Some notations. We write $\pi_{0}: \mathbf{R}^{m} \times \mathbf{R} \rightarrow \mathbf{R}^{m}:(x, z) \mapsto x$ respectively $\pi_{+}: \mathbf{R}^{m} \times \mathbf{R} \rightarrow \mathbf{R}:(x, z) \mapsto z$ for the projection on the "center" respectively stable direction. If $h(x, z)$ is a function on $\mathbf{R}^{m} \times \mathbf{R} \rightarrow \mathbf{R}$ we consider $\frac{1}{k !} \frac{\partial^{k} h}{\partial z^{k}}(x, 0) \cdot z^{k}$ if it exists; we then also denote

$$
|h|_{x, k}=\left|\frac{1}{k !} \frac{\partial^{k} h}{\partial z^{k}}(x, 0)\right|,
$$

and if $h$ takes values in $\mathbf{R}^{m} \times \mathbf{R}$ we abbreviate

$$
\left|\pi_{+} h\right|_{x, k}=:|h|_{+, k, x} .
$$

If $Y$ is a vector field we write $Y_{t}$ for its time $t$ map. This means that

$$
Y_{t}(v)=v+\int_{0}^{t} Y\left(Y_{s}(v)\right) d s .
$$

We fix $\tilde{c}>c>0$ where $\tilde{c}-c$ is 'as small as desired'. Using a cut off function we take care that the Lipschitz constant of $X$ in the $z$-direction is less than $\tilde{c}$, that is:

$$
\left|\pi_{+} X(x, z)-\pi_{+} X\left(x, z^{\prime}\right)\right| \leq \tilde{c}\left|z-z^{\prime}\right| .
$$

In the sequel of this induction step we shall neglect all objects of order $z^{k+1}$ and higher. For $w \in \mathbf{R}^{m} \times \mathbf{R}$ we want to estimate differences of the form

$$
\pi_{+}\left(X_{t}\left(w+\left(0, b(x) z^{k}\right)\right)-X_{t}(w)\right)=: d(t, x) z^{k}
$$

for some given function $b(x)$. We can write

$$
\pi_{+} X_{t}(w)=\pi_{+} w+\int_{0}^{t} \pi_{+} X\left(X_{s}(w)\right) d s
$$

and also

$$
\pi_{+} X_{t}\left(w+b(x) z^{k}\right)=\pi_{+} w+b(x) z^{k}+\int_{0}^{t} \pi_{+} X\left(X_{s}\left(w+b(x) z^{k}\right)\right) d s
$$

so

$$
\begin{aligned}
\pi_{+}\left(X_{t}\left(w+\left(0, b(x) z^{k}\right)\right)\right. & \left.-X_{t}(w)\right)=b(x) z^{k} \\
& +\int_{0}^{t}\left(\pi_{+} X\left(X_{s}\left(w+b(x) z^{k}\right)-\pi_{+} X\left(X_{s}(w)\right)\right) d s .\right.
\end{aligned}
$$


We use (6) and estimate (8), using the notation from (7) for $t>0$ :

$$
|d(t, x)||z|^{k} \leq|b(x)||z|^{k}+\int_{0}^{t} \tilde{c}|d(s, x)||z|^{k} d s .
$$

We see that $|d(t, x)|$ is bounded from above by the solution $\varphi(t)$ of the integral equation

$$
\varphi(t)=|b(x)|+\tilde{c} \int_{0}^{t} \varphi(s) d s
$$

or, if one prefers, the differential equation $\varphi^{\prime}=\tilde{c} \varphi$ with initial value $\varphi(0)=$ $|b(x)|$, which is known to be $\varphi(t)=|b(x)| e^{\tilde{c} t}$. We conclude that

$$
|d(t, x)| \leq|b(x)| e^{\tilde{c} t} .
$$

We trivially note that $\tilde{c}$ is also a Lipschitz constant for $\pi_{+}(-X)$. So also for $X_{-t}=(-X)_{t}$ we have the estimate

$$
\left|\pi_{+}\left(X_{-t}\left(w+\left(0, b(x) z^{k}\right)\right)-X_{-t}(w)\right)\right| \leq e^{\tilde{c} t}|b(x)||z|^{k} .
$$

Remark that $\pi_{0} \circ X_{-t} \circ X_{t}^{0}(x, z)=x$ for any $t$, since $X$ and $X^{0}$ have the same $\pi_{0}$-component. Next we want to study the limit $t \rightarrow \infty$ of $\pi_{+}\left(X_{-t} \circ X_{t}^{0}\right)(x, z)$, more specifically we want to consider the coefficient function of $z^{k}$. For that purpose we let $t_{1}>t_{2}>0, t=t_{1}-t_{2}$ and write, making use of (9):

$$
\begin{aligned}
\left|X_{-t_{1}} \circ X_{t_{1}}^{0}-X_{-t_{2}} \circ X_{t_{2}}^{0}\right|_{+, k, x} & =\left|X_{-t_{2}} \circ X_{-t} \circ X_{t_{1}}^{0}-X_{-t_{2}} \circ X_{t_{2}}^{0}\right|_{+, k, x} \\
& \leq e^{\tilde{c} t_{2}}\left|X_{-t} \circ X_{t_{1}}^{0}-X_{t_{2}}^{0}\right|_{+, k, x} \\
& =e^{\tilde{c} t_{2}}\left|X_{-t} \circ X_{t_{1}}^{0}-X_{-t}^{0} \circ X_{t_{1}}^{0}\right|_{+, k, x} .
\end{aligned}
$$

Lemma 2. We have for $t>0$ :

$$
\left|X_{t}-X_{t}^{0}\right|_{+, k, x} \leq e^{\tilde{c} t} \lim _{z \rightarrow 0} \frac{1}{|z|^{k}} \int_{0}^{t} e^{-\tilde{c} s}\left|\pi_{+} X^{1}\left(X_{s}^{0}(x, z)\right)\right| d s .
$$

Proof. We can write

$$
\begin{aligned}
X_{t}(w)-X_{t}^{0}(w)= & \int_{0}^{t}\left(X\left(X_{s}(w)\right)-X^{0}\left(X_{s}^{0}(w)\right)\right) d s \\
= & \int_{0}^{t}\left(X\left(X_{s}(w)\right)-X\left(X_{s}^{0}(w)\right)\right. \\
& \left.\quad+X\left(X_{s}^{0}(w)\right)-X^{0}\left(X_{s}^{0}(w)\right)\right) d s \\
= & \int_{0}^{t}\left(X\left(X_{s}(w)\right)-X\left(X_{s}^{0}(w)\right)+X^{1}\left(X_{s}^{0}(w)\right)\right) d s
\end{aligned}
$$

and so

$$
\begin{aligned}
\pi_{+}\left(X_{t}(w)-X_{t}^{0}(w)\right)=\int_{0}^{t}\left(\pi_{+} X\left(X_{s}(w)\right)-\pi_{+} X\left(X_{s}^{0}(w)\right)\right. & \\
& \left.+\pi_{+} X^{1}\left(X_{s}^{0}(w)\right)\right) d s
\end{aligned}
$$


Let us write

$$
\pi_{+}\left(X_{t}(w)-X_{t}^{0}(w)\right)=: d(t, x) \cdot z^{k}
$$

and also

$$
\psi(t)=\lim _{z \rightarrow 0} \frac{1}{|z|^{k}}\left|\pi_{+} X^{1}\left(X_{t}^{0}(w)\right)\right|
$$

Then for $t>0$ :

$$
|d(t, x)| \leq \tilde{c} \int_{0}^{t}|d(s, x)| d s+\int_{0}^{t} \psi(s) d s .
$$

We consider the differential equation

$$
\varphi^{\prime}(t)=\tilde{c} \varphi(t)+\psi(t)
$$

with initial value condition $\varphi(0)=0$. Its solution is $\varphi(t)=e^{\tilde{c} t} \int_{0}^{t} e^{-\tilde{c} s} \psi(s) d s$. We conclude that

$$
|d(t, x)| \leq e^{\tilde{c} t} \int_{0}^{t} e^{-\tilde{c} s} \psi(s) d s
$$

observe that the right hand sides of (14) and (11) are equal. This finishes the proof of lemma 2 .

Lemma 3. We have for $t>0$ :

$$
\left|X_{-t}-X_{-t}^{0}\right|_{+, k, x} \leq \lim _{z \rightarrow 0} \frac{1}{|z|^{k}} \int_{0}^{t} e^{\tilde{c} s} \mid \pi_{+}\left(X^{1}\left(X_{-t+s}^{0}(x, z)\right) \mid d s .\right.
$$

Proof. In the proof of lemma 2 we have only used that the Lipschitz constant of $\pi_{+} X$ in the $z$ direction is less than $\tilde{c}$. Since this is also true for the vector field $\pi_{+}(-X)$, and since $(-X)_{t}=X_{-t}$ we obtain

$$
\left|X_{-t}-X_{-t}^{0}\right|_{+, k, x} \leq e^{\tilde{c} t} \lim _{z \rightarrow 0} \frac{1}{|z|^{k}} \int_{0}^{t} e^{-\tilde{c} s}\left|\pi_{+} X^{1}\left(X_{-s}^{0}(x, z)\right)\right| d s .
$$

The integral in the right hand side of (16) can be rewritten as follows, using a substitution $s=t-\sigma$ :

$$
\begin{aligned}
\int_{0}^{t} e^{-\tilde{c} s}\left|\pi_{+} X^{1}\left(X_{-s}^{0}(x, z)\right)\right| d s & =\int_{t}^{0} e^{-\tilde{c}(t-\sigma)}\left|\pi_{+} X^{1}\left(X_{-t+\sigma}^{0}(x, z)\right)\right| d(-\sigma) \\
& =e^{-\tilde{c} t} \int_{0}^{t} e^{\tilde{c} \sigma}\left|\pi_{+} X^{1}\left(X_{-t+\sigma}^{0}(x, z)\right)\right| d \sigma
\end{aligned}
$$

If we insert (17) into (16) we get the desired right hand side in (15), modulo renaming $\sigma$ again $s$. This proves lemma 3 .

So now we continue the estimate in (10) as follows. We replace $(x, z)$ by $X_{t_{1}}^{0}(x, z)$ in (15) and obtain

$$
\begin{aligned}
\left|X_{-t_{1}} \circ X_{t_{1}}^{0}-X_{-t_{2}} \circ X_{t_{2}}^{0}\right|_{+, k, x} \\
\leq e^{\tilde{c} t_{2}} \lim _{z \rightarrow 0} \frac{1}{|z|^{k}} \int_{0}^{t} e^{\tilde{c} s}\left|\pi_{+}\left(X^{1}\left(X_{-t+s+t_{1}}^{0}(x, z)\right)\right)\right| d s \\
\quad=e^{\tilde{c} t_{2}} \lim _{z \rightarrow 0} \frac{1}{|z|^{k}} \int_{0}^{t} e^{\tilde{c} s}\left|\pi_{+}\left(X^{1}\left(X_{t_{2}+s}^{0}(x, z)\right)\right)\right| d s .
\end{aligned}
$$


Let us denote $X_{t_{2}+s}^{0}(x, z)=\left(x^{\prime}, z^{\prime}\right)$ then $\pi_{+} X^{1}\left(x^{\prime}, z^{\prime}\right)=G_{1}\left(x^{\prime}, z^{\prime}\right) \cdot\left(z^{\prime}\right)^{k}$ and hence $\left|\pi_{+} X^{1}\left(x^{\prime}, z^{\prime}\right)\right| \leq\left\|G_{1}\right\|_{\infty}\left|z^{\prime}\right|^{k}$. We can choose $\bar{c}$ close to $c$ such that $\left|z^{\prime}\right| \leq$ $e^{-\bar{c}\left(t_{2}+s\right)}|z|$. Hence

$$
\left|\pi_{+} X^{1}\left(x^{\prime}, z^{\prime}\right)\right| \leq\left\|G_{1}\right\|_{\infty} e^{-\bar{c}\left(t_{2}+s\right) k}|z|^{k}
$$

and so, plugging (19) into (18), we have:

$$
\begin{aligned}
\left|X_{-t_{1}} \circ X_{t_{1}}^{0}-X_{-t_{2}} \circ X_{t_{2}}^{0}\right|_{+, k, x} & \leq\left\|G_{1}\right\|_{\infty} e^{\tilde{c} t_{2}} \int_{0}^{t} e^{\tilde{c} s} e^{-\bar{c}\left(t_{2}+s\right) k} d s \\
& \leq\left\|G_{1}\right\|_{\infty} e^{(\tilde{c}-k \bar{c}) t_{2}} \int_{0}^{\infty} e^{(\tilde{c}-k \bar{c}) s} d s \\
& =\left\|G_{1}\right\|_{\infty} e^{(\tilde{c}-k \bar{c}) t_{2}} \frac{1}{k \bar{c}-\tilde{c}} .
\end{aligned}
$$

Since $\tilde{c}$ and $\bar{c}$ are close to $c$ we have that $\tilde{c}-k \bar{c}$ is close to $(1-k) c$. Since $k \geq 2$ and since $c>0$ we have that $\tilde{c}-k \bar{c}<0$. Hence, for $t_{2}$ sufficiently large the right hand side in (20) is as small as desired.

Using completeness of $\mathbf{R}$ we conclude that the coefficient of $z^{k}$ in $X_{-t}$ 。 $X_{t}^{0}(x, z)$ converges uniformly in $x$ for $t \rightarrow \infty$ to a continuous function say $\alpha(x)$ of $x$. Let us now explain why this limit has more smoothness.

Let us denote $\partial X_{t} / \partial x(x, z)=h(t, x, z)$. Then $h$ satisfies the equation

$$
\frac{\partial}{\partial t} h(t, x, z)=d X\left(X_{t}(x, z)\right) \cdot h(t, x, z)
$$

and if we write this down in components $\pi_{0} h=: h_{c}$ and $\pi_{+} h=: h_{+}$we consider thus the vector field

$$
\begin{aligned}
T X\left(x, z, h_{0}, h_{+}\right):=X(x, z)+ & \left(\frac{\partial \pi_{0} X}{\partial x}(x, z) \cdot h_{0}+\frac{\partial \pi_{0} X}{\partial z}(x, z) \cdot h_{+}\right) \frac{\partial}{\partial h_{0}} \\
& +\left(\frac{\partial \pi_{+} X}{\partial x}(x, z) h_{0}+\frac{\partial \pi_{+} X}{\partial z}(x, z) \cdot h_{+}\right) \frac{\partial}{\partial h_{+}} .
\end{aligned}
$$

Here we have used the concept of the 'tangent' of a map. This is, in general, defined as follows, see [AM78]. If $E, F$ and $G$ are, say, normed spaces and if $f$ : $U \subset E \rightarrow F$ is $C^{1}$ then we define $T f: U \times L_{c}(G, E) \rightarrow F \times L_{c}(G, F):(v, h) \mapsto$ $(f(v), d f(v) . h)$ The advantage of this concept is that it respects composition, that is: $T(g \circ f)=T g \circ T f$. Also the higher order chain rule becomes simply $T^{r}(g \circ f)=T^{r} g \circ T^{r} f$. We calculate

$$
\begin{aligned}
T X^{0}\left(x, z, h_{0}, h_{+}\right)=F(x) \frac{\partial}{\partial x}+G(x, 0) & z \frac{\partial}{\partial z}+\frac{\partial F}{\partial x}(x) h_{0} \frac{\partial}{\partial h_{0}} \\
& +\left(\frac{\partial G}{\partial x}(x, 0) \cdot z h_{0}+G(x, 0) h_{+}\right) \frac{\partial}{\partial h_{+}}
\end{aligned}
$$

and for $X=X^{1}$ :

$$
\begin{aligned}
& T X^{1}\left(x, z, h_{0}, h_{+}\right)=G_{1}(x, z) z^{k} \frac{\partial}{\partial z} \\
& +\left(\frac{\partial G_{1}}{\partial x}(x, z) z^{k} h_{0}+\frac{\partial G_{1}}{\partial z}(x, z) z^{k} h_{+}+k G_{1}(x, z) z^{k-1} h_{+}\right) \frac{\partial}{\partial h_{+}}
\end{aligned}
$$


By estimates of a similar kind as the ones above, and by using the flatness of $G_{1}$, the coefficient of $z^{h} h_{0}$ in the $h_{+}$-direction of $T\left(X_{-t_{1}} \circ X_{t_{1}}^{0}-X_{-t_{2}} \circ X_{t_{2}}^{0}\right)$ tends uniformly in $x$ to zero for $t_{2} \rightarrow \infty$. Hence, using completeness and a theorem of Weierstrass on interchanging limit and derivative, the function $\alpha(x)$ of $x$ is of class $C^{1}$.

This ends the induction step. So from now on we may assume that $X=$ $X^{0}+X^{1}$ where $X^{1}=O\left(|z|^{N}\right) \frac{\partial}{\partial z}$ where $N$ is 'as large as desired'. The next, and final, step is to eliminate $X^{1}$. Again we consider $X_{-t} \circ X_{t}^{0}$. The component in the $x$-direction is the identity. We examine the $z$-direction. Let $\kappa>0$ be a Lipschitz constant for $X$ and $X^{0}$ and let $t_{1}>t_{2}>0$. We can choose $C>0$ be so that $\left|X^{1}(x, z)\right| \leq C|z|^{N}$.

Then, for $t=t_{1}-t_{2}$, we have, in a similar way as in the estimates above (we hence skip details) :

$$
\begin{aligned}
\left|\pi_{+}\left(X_{-t_{1}} \circ X_{t_{1}}^{0}-X_{-t_{2}} \circ X_{t_{2}}^{0}\right)(x, z)\right| & \leq e^{\kappa t_{2}} \int_{0}^{t} e^{\kappa s}\left|X^{1}\left(X_{t_{2}+s}^{0}(x, z)\right)\right| d s \\
& \leq C e^{\kappa t_{2}} \int_{0}^{t} e^{\kappa s}\left|\pi_{+} X_{t_{2}+s}^{0}(x, z)\right|^{N} d s \\
& \leq C|z|^{N} e^{\kappa t_{2}} \int_{0}^{t} e^{\kappa s} e^{-\tilde{c}\left(t_{2}+s\right) N} d s \\
& \leq C|z|^{N} \frac{e^{(\kappa-\tilde{c} N) t_{2}}}{\tilde{c} N-\kappa}
\end{aligned}
$$

Hence, provided that $N>\kappa / \tilde{c}$, we have that the limit for $t \rightarrow \infty$ of $X_{-t} \circ X_{t}^{0}$ converges uniformly in $(x, z)$ to a continuous $H(x, z)$. In order to show that $H$ is finitely smooth we can, again, pass to the tangent map $T\left(X_{-t} \circ X_{t}^{0}\right)$; a computation shows that we can make similar estimates as above in (21) for the coefficients of $h$, provided that we replace $N$ by $N-1$.

This finishes the proof of Theorem 3.

\section{Proof of Theorem 1}

Before starting the actual proof, let us first give an outline. A family of invariant manifolds $z=\varphi(x, y, \varepsilon, \lambda)$ of (1) is expressed by a solution to the partial differential equation

$$
\varepsilon^{r} m(x, y)\left(-x \frac{\partial \varphi}{\partial x}+q_{1} y_{1} \frac{\partial \varphi}{\partial y_{1}}+\cdots+q_{n} y_{n} \frac{\partial \varphi}{\partial y_{n}}\right)=-b(\lambda) \varphi+f(x, y, \varphi, \varepsilon, \lambda),
$$

which we can try to solve using the method of characteristics. Given a suitable initial curve $\gamma$, we will follow orbits of the system

$$
\left\{\begin{array}{rl}
\dot{x} & =-x \varepsilon^{r} m(x, y) \\
\dot{y}_{i} & =q_{i} y_{i} \varepsilon^{r} m(x, y),
\end{array} \quad i=1, \ldots, n,\right.
$$

which are characteristics for the PDE. The value of the center manifold at a given point $\left(x_{*}, y_{*}, \varepsilon_{*}, \lambda\right)$ is hence determined by an integral along this characteristic. Integration along these characteristics is done in local $C^{k}$-normal forms, where 
we find explicit expressions for the value of the invariant manifold. If we obtain that a given invariant manifold is $C^{k}$ in local $C^{k}$-normal form coordinates, and if we can do this for any $k$, then this proves the $C^{\infty}$ smoothness.

Let us now do some preparatory work. Using the implicit function theorem, we find a local graph $z=\zeta(x, y, \varepsilon, \lambda)$ where $\dot{z}=0$ in (1). Subtracting $\zeta$ from $z$, it is clear that, without loss of generality, we may assume that

$$
f(x, y, 0, \varepsilon, \lambda)=O\left(\varepsilon^{r}\right) .
$$

This way, vector field (1) has, for $\varepsilon=0$, a (hyper)plane of singular points given by $z=0$. Similarly, we can assume that

$$
f(x, y, 0, \varepsilon, \lambda)=0, \quad \forall(x, y) \text { for which } m(x, y)=0 .
$$

At each point of $\{\varepsilon=z=0\}$, we have the existence of $C^{k}$-center manifolds, and even the existence of a $C^{k}$-conjugation that normally linearizes (1) at that point. In case $m \neq \equiv \pm 1$, we also have one or more subspaces of singular points given by $z=m(x, y)=0$.

Lemma 4. Let the family of vector fields $X_{\varepsilon, \lambda}$ be as in (1), with properties (22) and (23). Near each point $(x, y, z, \varepsilon)=\left(x_{0}, y_{0}, 0,0\right)$ for which holds

$$
b(\lambda)+\frac{\partial}{\partial z} f\left(x_{0}, y_{0}, 0,0, \lambda\right)>0
$$

and for each $k \geq 0$ there exists a $C^{k}$ conjugacy of the form

$$
(x, y, z) \mapsto(x, y, \tilde{z}) ; \quad \tilde{z}=\Psi_{k}(x, y, z, \varepsilon, \lambda)
$$

(with $\frac{\partial \Psi_{k}}{\partial z}(x, y, 0, \varepsilon, \lambda)=1$ ) conjugating (1) to a family of vector fields of the form

$$
X_{\varepsilon, \lambda}:\left\{\begin{array}{rl}
\dot{x} & =-x \varepsilon^{r} m(x, y) \\
\dot{y_{i}} & =q_{i} y_{i} \varepsilon^{r} m(x, y), \\
\dot{z} & =-b_{k}(x, y, \varepsilon, \lambda) z,
\end{array} \quad i=1, \ldots, n,\right.
$$

for some $b_{k}>0$. In case $m \not \equiv \pm 1$, the lemma also holds at points $(x, y, z, \varepsilon)=$ $\left(x_{0}, y_{0}, 0, \varepsilon_{0}\right)$ for which $m\left(x_{0}, y_{0}\right)=0$ and $b(\lambda)+\frac{\partial}{\partial z} f\left(0, y_{0}, 0, \varepsilon_{0}, \lambda\right)>0$.

Proof. We first consider a $C^{k} \lambda$-family of center manifold of $X_{\varepsilon, \lambda}+0 \frac{\partial}{\partial \varepsilon}$ near $(x, y, z, \varepsilon)=\left(x_{0}, y_{0}, 0,0\right)$. After straightening a sufficiently smooth center manifold, this brings the family in the form of Theorem 3, after which one can apply a local normal linearization.

Without loss of generality, we may assume that the $C^{1}$-normal form from Lemma 4, applied at $\left(x_{0}, y_{0}\right)=(0,0)$, is valid for $x, y_{i}, z \in[-1,1], \varepsilon \in[0,1]$, $\lambda \in \Lambda$, and that $b_{1}(x, y, \varepsilon, \lambda) \geq \delta$ for some $\delta>0$ on this domain. Let us now construct a center manifold with the required smoothness properties.

Recall the function

$$
m(x, y)= \pm x^{\kappa_{0}} y_{1}^{\kappa_{1}} \ldots y_{n}^{\kappa_{n}},
$$


and observe that it divides the space into two parts: a part where $m(x, y)>0$, and a part where $m(x, y)<0$. We will construct, independently for each part, a smooth center manifold $z=\varphi(x, y, \varepsilon, \lambda)$, and show that it extends to a smooth manifold also at points where $m(x, y)=0$. More specifically, in the region $m(x, y)>0$, we will construct the center manifold by following orbits in positive time, starting from $|x|=1$. In the region $m(x, y)<0$, we will construct the center manifold by following orbits in positive time, starting from $\|y\|=1$, see later.

The construction in each part of the space can be done completely independently: extending to $(x, y)$ with $m(x, y)=0$ can always be done without any problem, as will be clear from the following Lemma:

Lemma 5. Let $k$ be an arbitrary integer or $\infty$. Let $U_{1}, \ldots, U_{p}$ be the connected components of $[-1,1]^{n+1} \backslash\left\{m^{-1}(0)\right\}$. Suppose that $z=\varphi(x, y, \varepsilon, \lambda)$ represents an invariant manifold of (1) with the property that $\varphi(x, y, \varepsilon, \lambda)=0$ when $m(x, y)=$ 0 , and that each $\varphi_{i}=\left.\varphi\right|_{\overline{U_{i}}}$ is $C^{k}$. Then $\varphi$ is $C^{k}$.

Proof. Suppose first that $\kappa_{0} \neq 0$. Then some components $U_{\ell}$ and $U_{j}$ have a partly shared boundary on $\{x=0\}$. We show that $\varphi$ is $C^{k}$ along $\{x=$ $0\} \cap \overline{U_{\ell}} \cap \overline{U_{j}}$. To that end, notice that there is a unique formal power series expansion $z=\hat{\varphi}:=\sum_{i=1}^{\infty} \varphi_{i}(y, \varepsilon, \lambda) x^{k}$ with

$$
\begin{array}{r}
\hat{\varphi}=\frac{1}{b(\lambda)}\left(\varepsilon^{r} m(x, y)\left(-x \frac{\partial \hat{\varphi}}{\partial x}+q_{1} y_{1} \frac{\partial \hat{\varphi}}{\partial y_{1}}+\cdots+q_{n} y_{n} \frac{\partial \hat{\varphi}}{\partial x}\right)\right. \\
-f(x, y, \hat{\varphi}, \varepsilon, \lambda)) .
\end{array}
$$

This is easily seen by an induction argument, observing that knowledge of $\hat{\varphi}$ up to a given degree $N$ in $x$ completely determines the right-hand side of the above equation up to at least degree $N+1$ in $x$. Here, we have used that $f(x, y, 0, \varepsilon, \lambda)=O(m(x, y))$, see $(23)$. Since both $\varphi_{j}$ and $\varphi_{\ell}$ are invariant manifolds, their order $k$ Taylor expansion satisfies the above equation, and therefore their order $k$ Taylor expansion coincide.. This shows the $C^{k}$-smoothness along $\{x=0\} \cap \overline{U_{\ell}} \cap \overline{U_{j}}$. Similar arguments show the $C^{k}$-smoothness along $\left\{y_{i}=0\right\} \cap \overline{U_{\ell}} \cap \overline{U_{j}}$ when $\kappa_{i} \neq 0$.

Center manifold in $\{m(x, y) \geq 0, x \geq 0\}$

Let us first deal with the region $m(x, y) \geq 0, x \geq 0$. The region $\{m(x, y) \geq$ $0, x \leq 0\}$ is treated similarly (in the cases where this domain is not empty), after applying the transformation $x \mapsto-x$. We show that the center manifold $z=\varphi(x, y, \varepsilon, \lambda)$, defined for $(x, y)$ with $m(x, y)>0$ and $x>0$, has a smooth extension towards the boundary $\{x=0\} \cup\{m(x, y)=0\}$.

Define

$$
\gamma=\{x=1, z=0,\|y\|<1, \varepsilon \in[0,1], m(x, y) \geq 0\} .
$$

We aim to find the unique center manifold, defined on $m(x, y) \geq 0$, that contains the set $\gamma$. We consider orbits through $\gamma$ and write the union as a $(\varepsilon, \lambda)$-family of graphs $z=\varphi(x, y, \varepsilon, \lambda)$. 
Consider the vector field (1) in $C^{1}$ normal form coordinates, as given in Lemma 4 (applied at $\left.\left(x_{0}, y_{0}\right)=(0,0)\right)$. The section $\gamma$ is given in these coordinates as

$$
\tilde{\gamma}=\left\{x=1, \tilde{z}=\Psi_{1}(x, y, 0, \varepsilon, \lambda),\|y\|<1, \varepsilon \in[0,1], m(x, y) \geq 0\right\} .
$$

Due to its elementary form, a center manifold of the vector field in normal form, containing $\tilde{\gamma}$, can be written explicitly as a graph $z=\tilde{\varphi}(x, y, \varepsilon, \lambda)$ : for $0<x \leq 1,\|y\|<1$ and for $\varepsilon>0$ we write

$$
\tilde{\varphi}(x, y, \varepsilon, \lambda):=\Psi_{1}\left(1, x^{q} y, 0, \varepsilon, \lambda\right) \exp \left(\frac{-1}{\varepsilon^{r} m(x, y) x^{\kappa q-\kappa_{0}}} \int_{x}^{1} \frac{B(s, x, y, \varepsilon, \lambda)}{s^{1+\kappa_{0}-\kappa q}} d s\right)
$$

where $x^{q} y$ is a shortcut for $\left(x^{q_{1}} y_{1}, \ldots, x^{q_{n}} y_{n}\right), \kappa q$ is a shortcut for $\kappa_{1} q_{1}+\cdots+$ $\kappa_{n} q_{n}$, and where

$$
B(s, x, y, \varepsilon, \lambda):=b_{1}\left(s,(x / s)^{q} y, \varepsilon, \lambda\right) .
$$

It is not hard to see that as long as $x \varepsilon m(x, y) \neq 0$ the graph $\tilde{z}=\tilde{\varphi}(x, y, \varepsilon, \lambda)$ is a $C^{1}$ invariant manifold of the vector field in $C^{1}$-normal form. Let us look at the boundary of this domain, i.e. at $x \varepsilon m(x, y)=0$. To that end, we give an elementary bound of the exponential appearing in $\tilde{\varphi}$ :

Lemma 6. Given any $N$, and any $0<x_{0}<1$ there exists $\varepsilon_{N}>0$ for which

$$
\exp \left(\frac{-1}{\varepsilon^{r} m(x, y) x^{\kappa q-\kappa_{0}}} \int_{x}^{1} \frac{B(s, x, y, \varepsilon, \lambda)}{s^{1+\kappa_{0}-\kappa q}} d s\right) \leq C_{N}(x \varepsilon m(x, y))^{N}
$$

for some $C_{N}>0$, as long as $\varepsilon \leq \varepsilon_{N}$ and $0<|x|<\left|x_{0}\right|$. When $m \neq 1$, we may choose $\varepsilon_{N}=1$.

Note: the case $m \equiv-1$ does not occur here, since $m$ is assumed positive in this section.

Proof. We have $B(s, x, y, \varepsilon, \lambda) \geq \delta$, and write $K=\kappa_{0}-\kappa q$. We distinguish three cases: $K=0, K>0$ and $K<0$. When $K=0$, we find

$$
\int_{x}^{1} B(s, x, y, \varepsilon, \lambda) \frac{d s}{s} \geq \delta \int_{x}^{1} \frac{d s}{s}=\delta|\ln x|,
$$

so the exponential under study is bounded by $\exp \frac{-\delta|\ln x|}{\varepsilon^{r} m(x, y)}$. When $K=0$, we have $\kappa_{0}=\kappa q$, so the property $m \equiv 1$ is equivalent to the property $\kappa_{0}=0$. When $m \not \equiv 1$ (and hence $\kappa_{0} \geq 1$ ), we can use $|\ln x| \geq\left|\ln x_{0}\right|$ and $m(x, y) \leq$ $x^{1 / 2} m(x, y)^{1 / 2}$ to find that the exponential is bounded by

$$
\exp \frac{-\delta\left|\ln x_{0}\right|}{\varepsilon^{r} x^{1 / 2} m(x, y)^{1 / 2}} \leq \frac{(2 N) !}{\left(\delta\left|\ln x_{0}\right|\right)^{2 N}}\left(\varepsilon^{r} x^{1 / 2} m(x, y)^{1 / 2}\right)^{2 N} \leq C_{N}(x \varepsilon m)^{N} .
$$

On the other hand, when $m \equiv 1$, we find that the exponential is bounded by

$$
\begin{aligned}
\exp \frac{-\delta|\ln x|}{\varepsilon^{r}} & \leq \exp \frac{-\delta\left|\ln x_{0}\right|}{2 \varepsilon^{r}} \cdot \exp \frac{-\delta|\ln x|}{2 \varepsilon^{r}} \\
& \leq \frac{N !}{\left(\frac{\delta}{2}\left|\ln x_{0}\right|\right)^{N}} \varepsilon^{r N} \cdot x^{\delta / 2 \varepsilon^{r}} \leq \frac{N !}{\left(\frac{\delta}{2}\left|\ln x_{0}\right|\right)^{N}}\left(\varepsilon^{r} x\right)^{N} \leq C_{N}(x \varepsilon m)^{N}
\end{aligned}
$$


as soon as $\varepsilon^{r} \leq \frac{\delta}{2 N}$. This illustrates the necessity of bounding $\varepsilon$ by some $\varepsilon_{N}$ in case $m \equiv 1$. Let us now deal with the case $K<0$. Then

$$
\int_{x}^{1} B(s, x, y, \varepsilon, \lambda) \frac{d s}{s^{1+K}} \geq \delta \int_{x}^{1} \frac{d s}{s^{1+K}}=\frac{\delta}{|K|}\left(1-x^{|K|}\right) \geq \frac{\delta}{|K|}\left(1-x_{0}^{|K|}\right) .
$$

The exponential is then bounded by

$$
\exp \left(-\frac{\delta}{\varepsilon^{r} m(x, y) x^{|K|}} \frac{1-x_{0}^{|K|}}{|K|}\right) .
$$

Like before, it is easily shown that such an expression is $O(x \varepsilon m)^{N}$ for any $N$. Finally assume $K>0$ :

$$
\int_{x}^{1} B(s, x, y, \varepsilon, \lambda) \frac{d s}{s^{1+K}} \geq \delta \int_{x}^{1} \frac{d s}{s^{1+K}}=-\frac{\delta}{K} \frac{x^{K}-1}{x^{K}} \leq-\frac{\delta}{K} \frac{x_{0}^{K}-1}{x^{K}} .
$$

It follows that the exponential under consideration is bounded by

$$
\exp \left(-\frac{\delta}{\varepsilon^{r} m(x, y)} \frac{1-x_{0}^{K}}{K}\right) .
$$

Like before, it follows that such an expression is $O\left(\varepsilon^{N} m^{2 N}\right)$ for any $N$. Let us conclude by showing that $m(x, y)=O(x)$, i.e. that $\kappa_{0} \neq 0$ in this case. This is clear since $K=\kappa_{0}-\kappa q>0$.

Using Lemma 6 , it is easy to show that $\tilde{\varphi}$ extends to $x \varepsilon m(x, y)=0, x \geq 0$ in a $C^{1}$-way. Note: the smoothness is not guaranteed at $(x, \varepsilon)=( \pm 1,0)$, but this is not essential.

In original coordinates, the manifold is a graph

$$
z=\varphi(x, y, \varepsilon, \lambda)
$$

defined for $x \in[0,1],\|y\|<1, \varepsilon \in[0,1]$ and $\lambda \in \Lambda$ (keeping $(x, \varepsilon) \neq(1,0)$.) We show that for any given $k$ this given manifold is $C^{k}$, provided we restrict $\varepsilon$ to a $k$-dependent interval.

First observe that the manifold consists of regular orbits departing from the sections $\gamma_{ \pm}$. This implies that for $x \varepsilon m \neq 0$ it is immediately clear that $\varphi$ is $C^{\infty}$. Let us now discuss the smoothness outside $x=0$ :

Lemma 7. The $(\varepsilon, \lambda)$-family of invariant manifolds $z=\varphi(x, y, \varepsilon, \lambda)$ is $C^{\infty}$ in $\{x>0\}$.

Proof. We only need to deal with the smoothness at $\varepsilon m=0$, so take a specific $\left(x_{*}, y_{*}, \varepsilon_{*}\right)$, with $\varepsilon_{*} m\left(x_{*}, y_{*}\right)=0$ and with $x_{*}>0$. We will show that for any given integer $k$, the function $\varphi$ is $C^{k}$ at $\left(x_{*}, y_{*}, \varepsilon_{*}, \lambda\right)$. Recall that $\varphi\left(x_{*}, y_{*}, \varepsilon_{*}, \lambda\right)=0$.

The orbit of

$$
\left\{\begin{array}{rl}
\dot{x} & =-x \\
\dot{y}_{i} & =q_{i} y_{i},
\end{array} \quad i=1, \ldots, n,\right.
$$

through $\left(x_{*}, y_{*}\right)$ reaches $\{x=1\}$ in finite (negative) time in a point $(1, \tilde{y})$, with $\tilde{y}_{i}=\frac{y_{* i}}{x_{*}^{q_{i}}}$; let us denote by $\gamma_{x_{*}, y_{*}}$ the orbit-segment between $x=x_{*}$ and $x=1$. 

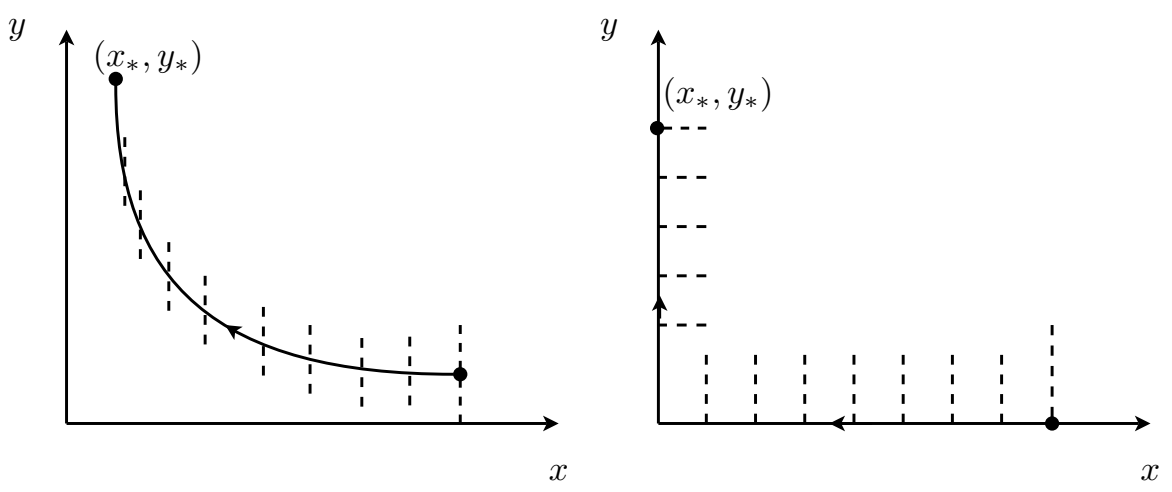

Figure 1: The orbit through $\left(x_{*}, y_{*}\right)$ is divided in several pieces. To the left the case $x_{*}>0$ (treated in Lemma 7 ), to the right the case $x_{*}=0$ (treated in Lemma 8).

Because $\varepsilon m=0$ at the end point of $\gamma_{x_{*}, y_{*}}$, we have $\varepsilon m=0$ on the whole of $\gamma_{x_{*}, y_{*}}$ (either $\varepsilon_{*}=0$ in which case this is trivially true, or $m\left(x_{*}, y_{*}\right)=0$, in which case some $y_{* i}=0$, hence $y_{i}=0$ on the orbit). This shows that one can apply Lemma 4 at all points $\left(x_{0}, y_{0}, 0, \varepsilon_{*}\right)$, where $\left(x_{0}, y_{0}\right)$ lies on $\gamma_{x_{*}, y_{*}}$. This way, one obtains a finite covering of $\left\{(x, y, z):(x, y) \in \gamma_{x_{*}, y_{*}}, z=0\right\}$ by neighborhoods in which we have $C^{k}$-normal forms for (1). There is a value $\bar{\varepsilon}_{k}$ so that all these normal forms are valid for $\varepsilon \in\left[0, \bar{\varepsilon}_{k}\right]$. We also observe that the center manifold is $O(\varepsilon m)$, so keeping $|\varepsilon m|$ close enough to 0 , the center manifold $z=\varphi(x, y, \varepsilon, \lambda)$ is entirely overlapped by such neighborhoods. Let us next treat the $C^{k}$-smoothness of $\varphi$ near a given point $(x, y, \varepsilon)=\left(x_{*}, y_{*}, \varepsilon_{*}\right)$, with $x_{*}>0$. On the orbit $\gamma_{x_{*}, y_{*}}$, we can choose a number of points $\left(p_{i}\right)_{i=1, \ldots, N}$, with $p_{i}=\left(x_{(i)}, y_{(i)}\right), p_{1}=(1, \widetilde{y})$ and $p_{N}=\left(x_{*}, y_{*}\right)$, so that the piece of the orbit between $p_{i}$ and $p_{i+1}$ is entirely visible in a single neighborhood $V_{i}$ with a $C^{k_{-}}$ normal form, see Figure 1. Now, the value of $p_{2}$ is determined by integrating an orbit through $\gamma$. Below, we will prove that the value of $\varphi$ is $C^{k}$-smooth near $p_{2}$, but let us first indicate how the lemma is an immediate consequence of such a proof. Once the smoothness near any $p_{2}$ is shown, we can define a $C^{k}$-manifold

$$
\gamma_{(2)}=\left\{x=x_{(2)},\left\|y-y_{(2)}\right\| \ll 1, z=\varphi(x, y, \varepsilon, \lambda),|m \varepsilon| \ll 1\right\},
$$

through $p_{2}$. The value of $p_{3}$ can now be determined by integrating an orbit from the manifold $\gamma_{(2)}$, in a way identical to the way the value of $p_{2}$ is obtained by integrating from $\gamma$. By repeating the same argument, we show that $\varphi$ is smooth near $p_{3}$. Continuing by induction, we finally find the smoothness of $\varphi$ near $p_{N}=\left(x_{*}, y_{*}\right)$, and for $\varepsilon=\varepsilon_{*}$. Let us now show the smoothness of $\varphi$ near any $p_{i}$, using the normal form

$$
\left\{\begin{array}{rl}
\dot{x} & =-x \varepsilon^{r} m(x, y) \\
\dot{y}_{i} & =q_{i} y_{i} \varepsilon^{r} m(x, y), \\
\dot{\tilde{z}} & =-b_{k}(x, y, \varepsilon, \lambda) \tilde{z},
\end{array} \quad i=1, \ldots, n,\right.
$$

The initial manifold $\gamma$ is seen in normal form coordinates as a manifold

$$
\tilde{\gamma}=\left\{x=1,\|y\|<1, \tilde{z}=\Psi_{k}\left(1, y, z_{0}(1, y, \varepsilon, \lambda), \varepsilon, \lambda\right), \varepsilon \in\left[0, \bar{\varepsilon}_{k}\right]\right\}
$$


for some $C^{k}$ function $z_{0}(1, y, \varepsilon, \lambda)$, where $\Psi_{k}$ is like in Lemma 4 (and for $p_{i}=p_{2}$ we have $\left.z_{0} \equiv 0\right)$. We hence find an invariant manifold $\tilde{z}=\tilde{\varphi}(x, y, \varepsilon, \lambda)$ containing $\tilde{\gamma}$, where

$$
\begin{aligned}
\tilde{\varphi}=\Psi_{k}\left(1, x^{q} y, z_{0}\left(1, x^{q} y, \varepsilon, \lambda\right), \varepsilon, \lambda\right) . \\
\quad \exp \left(\frac{-1}{\varepsilon^{r} m x^{\kappa q-\kappa_{0}}} \int_{x}^{1} \frac{B_{k}(s, x, y, \varepsilon, \lambda)}{s^{1+\kappa_{0}-\kappa q}} d s\right) .
\end{aligned}
$$

Here $B_{k}(s, x, y, \varepsilon, \lambda)=\tilde{b}_{k}\left(s,(x / s)^{q} y, \varepsilon, \lambda\right)$, and the smoothness of $\tilde{\varphi}$ is shown easily: with each partial derivative of $\tilde{\varphi}$, the exponential can be factored out, and using Lemma 6 , we find that such an exponential is sufficiently flat w.r.t. $\varepsilon m$ as $\varepsilon m \rightarrow 0$. This shows that $\tilde{\varphi}$ has a $C^{k}$-extension to those points where $\varepsilon m=0$.

The technique of proof can be adapted to prove the smoothness at points where $x=0$. However, one has to keep in mind that one has to work with a singular orbit, like in Figure 1. One needs to cover this singular path by a finite number of neighborhoods in which normal forms are taken. On the $x$-axis, one may proceed like in the proof of Lemma 7 . Once one obtains the smoothness near the origin, one can proceed along $\{x=0\}$. Here we distinguish two cases. When $m=O(x)$, we can cover the plane $\{x=0\}$ by neighborhoods where we take normal forms like in Lemma 4 . When $m \neq O(x)$, the set $\{x=0\}$ is nonsingular (outside $m \varepsilon=0$ ). The characteristics are hence regular curves.

Lemma 8. Given $k \geq 1$, the function $\varphi(x, y, \varepsilon, \lambda)$, defined for $x \geq 0$, is $C^{k}$ along $x=0$, for $\varepsilon$ sufficiently small. When $m \neq 1$, the function $\varphi(x, y, \varepsilon, \lambda)$, defined for $x \geq 0$, is $C^{\infty}$ along $x=0$, for $\varepsilon$ sufficiently small.

Proof. We first deal with the smoothness near the origin $(x, y)=(0,0)$, and then show how the smoothness properties can be extended throughout the rest of $\{x=0\}$.

Given $k$, we consider a $C^{k}$-local normal form at $\left(x_{0}, y_{0}, \varepsilon\right)=(0,0,0)$. For $\nu$ small enough, the section $\{x=\nu,\|y\|<\nu, \varepsilon<\nu\}$ is visible in this normal form, and along that segment we already have established the smoothness of the center manifold. In local coordinates, this manifold is given by $\tilde{z}=\tilde{\varphi}(x, y, \varepsilon, \lambda)$, and we hence find

$$
\tilde{\varphi}(x, y, \varepsilon, \lambda)=\tilde{\varphi}\left(\nu,(x / \nu)^{q} y, \varepsilon, \lambda\right) \exp \left(-\frac{1}{\varepsilon^{r} m x^{\kappa q-\kappa_{0}}} \int_{x}^{\nu} \frac{B_{k}(s, x, y, \varepsilon, \lambda)}{s^{1+\kappa_{0}-\kappa q}} d s\right),
$$

with $B_{k}(s, x, y, \varepsilon, \lambda)=\tilde{b}_{k}\left(s,(x / s)^{q} y, \varepsilon, \lambda\right)$. From this expression, it is clear that $\tilde{\varphi}$ is $C^{k}$-smooth near $(x, y, \varepsilon)=(0,0,0)$. The smoothness along $x=0$ in particular follows from the estimates in Lemma 6 . We can repeat this construction for any $k$, restricting $\varepsilon$ to a $k$-dependent neighborhood of 0 . However, when $m \not \equiv 1$, we can repeat the construction by taking local normal forms at $\left(x_{0}, y_{0}, \varepsilon\right)=(0,0, \varepsilon)$ with nonzero $\varepsilon$ (because then $(x, y, z, \varepsilon)=(0,0,0, \varepsilon)$ is also a semi-hyperbolic singularity). This observation suffices to see that the center manifold is $C^{\infty}$ near the origin when $m \not \equiv 1$.

Let us now deal with the smoothness of the manifold $z=\varphi(x, y, \varepsilon, \lambda)$ in the rest of $\{x=0\}$. At points of this plane where $\varepsilon m(x, y) \neq 0$, we can draw characteristics that reach a neighborhood of the origin. Since such characteristics 
are regular, the smoothness of the center manifold at such points follows from the smoothness of the center manifold near the origin. So it suffices to consider those points of $\{x=0\}$ with $\varepsilon m=0$. We can cover this part of $\{x=0\}$ by a finite number of neighborhoods where we have $C^{k}$-normal forms as in Lemma 4 (given a fixed $k$ ).

Given a fixed $\left(x_{*}, y_{*}, \varepsilon_{*}\right)$ with $x_{*}=0$ and $\varepsilon_{*} m\left(x_{*}, y_{*}\right)=0$. We may suppose $y_{*} \neq 0$. The characteristic through $\left(x_{*}, y_{*}\right)$ travels through a finite number of neighborhoods where we have normal forms. Let us introduce the variable

$$
\rho=R(y):=\sqrt{y_{1}^{2 q^{1}}+\cdots+y_{n}^{2 q^{n}}}, \quad q^{i}:=\frac{q_{1} q_{2} \cdots q_{n}}{q_{i}} .
$$

We will treat the passage from a section $R(y)=\nu$ to a section $R(y)=\mu$ (with $0<\nu<\mu$ ), and show that the smoothness properties in the first section are transported to the second section near the given characteristic. Since the characteristic is cut by a finite number of such sections $R(y)=$ const, we obtain the required smoothness at $\left(x_{*}, y_{*}, \varepsilon_{*}\right)$ by an induction argument. We remark that the characteristic through $\left(x_{*}, y_{*}\right)$ tends towards the origin in negative time, so for the initial induction step one can rely on the smoothness results obtained at the beginning of the proof of this lemma, i.e. the smoothness near the origin. So let us focus on a single passage between $R(y)=\nu$ to $R(y)=\mu$. We remark that if we write $\rho=R(y)$, then

$$
\dot{\rho}=Q \rho \varepsilon^{r} m(x, y), \quad \text { with } Q:=n q_{1} \ldots q_{n} .
$$

We use $\rho$ as a variable to parameterize the characteristic. Suppose in local $C^{k}$-coordinates, the center manifold $\tilde{z}=\tilde{\varphi}(x, y, \varepsilon, \lambda)$ is shown to be $C^{k}$-smooth near a given point on the section $R(y)=\nu$. Given a point $(x, y)$ on $R(y)=\mu$, its characteristic meets $R(y)=\nu$ at a point which we denote

$$
(\tilde{x}, \tilde{y})=\left(x(\mu / \nu)^{1 / Q}, y_{1}(\nu / \mu)^{q_{1} / Q}, \ldots, y_{n}(\nu / \mu)^{q_{n} / Q}\right) .
$$

Using this notation, we have

$$
\begin{aligned}
& \tilde{\varphi}(x, y, \varepsilon, \lambda)=\tilde{\varphi}(\tilde{x}, \tilde{y}, \varepsilon, \lambda) \\
& \quad \exp \left(\frac{-1}{Q \varepsilon^{r} m(x, y)} \int_{\nu}^{\mu} \frac{B_{k}(\rho, x, y, \varepsilon, \lambda)}{\rho \cdot(\rho / \nu)^{\left(\kappa_{1} q_{1}+\cdots+\kappa_{n} q_{n}-\kappa_{0}\right) / Q}} d \rho\right) .
\end{aligned}
$$

where $B_{k}(\rho, x, y, \varepsilon, \lambda)=\tilde{b}_{k}\left(x(\rho / \nu)^{1 / Q}, y_{1}(\nu / \rho)^{q_{1} / Q}, \ldots, y_{n}(\nu / \rho)^{q_{n} / Q}, \varepsilon, \lambda\right)$.

This expression seems complicated at first sight, but the integral inside it is completely regular (since we keep $\rho$ outside 0 here), and can be bounded from below by a positive constant. Regardless of whether $m \equiv 1$ or not, it is easily seen that this expression is $C^{k}$ smooth at $x=0$ and at points where $\varepsilon m=0$.

Center manifold in $\{m(x, y) \leq 0\}$

When $m$ is negative, we use a slightly different approach: where the center manifold was constructed by following orbits through $\{x= \pm 1\}$ in the domain $\{m \geq 0\}$, we will now construct a center manifold by putting an initial condition on the sphere $R(y)=1$. Here, we define

$$
R(y):=\sqrt{y_{1}^{2 q^{1}}+\cdots+y_{n}^{2 q^{n}}}, \quad q^{i}:=\frac{q_{1} q_{2} \cdots q_{n}}{q_{i}} .
$$


We integrate from the sphere

$$
C: y_{1}^{2 q^{1}}+\cdots+y_{n}^{2 q^{n}}=1 .
$$

It can be readily verified that, if we introduce the variable $\rho=R(y)$, then

$$
\dot{\rho}=Q \rho \varepsilon^{r} m(x, y) ; \quad \text { with } Q:=n . q_{1} \ldots q_{n} .
$$

We recall that the $C^{1}$-normal form from Lemma 4 , applied at $\left(x_{0}, y_{0}\right)=(0,0)$, is valid for $x, y_{i}, z \in[-1,1], \varepsilon \in[0,1], \lambda \in \Lambda$. We assume that $b_{1}(x, y, \varepsilon, \lambda) \geq \delta$ for some $\delta>0$ on this domain.

Let $\gamma=\{y \in C, z=0,|x| \leq 1, \varepsilon \in[0,1], m(x, y) \leq 0\}$. We find an invariant manifold by considering all orbits through $\gamma$. In the $C^{1}$-normal form, this initial plane is seen as an initial manifold

$$
\tilde{\gamma}=\left\{y \in C, \tilde{z}=\Psi_{1}(x, y, 0, \varepsilon, \lambda),|x| \leq 1, \varepsilon \in[0,1], m(x, y) \leq 0\right\} .
$$

We now write, for $m<0, \varepsilon>0$ and $y \neq 0$,

$$
\begin{aligned}
& \tilde{\varphi}(x, y, \varepsilon, \lambda)=\Psi_{1}\left(x \rho^{1 / Q}, y_{1} / \rho^{q_{1} / Q}, \ldots, y_{n} / \rho^{q_{n} / Q}, 0, \varepsilon, \lambda\right) . \\
& \exp \left(\frac{-1}{Q \varepsilon^{r}|m(x, y)| \rho^{\left(\kappa_{0}-\kappa_{1} q_{1}-\cdots-\kappa_{n} q_{n}\right) / Q}} \int_{\rho}^{1} \frac{B(s, x, y, \varepsilon, \lambda)}{s^{1+\left(\kappa_{1} q_{1}+\cdots+\kappa_{n} q_{n}-\kappa_{0}\right) / Q}} d s\right),
\end{aligned}
$$

evaluating the right-hand side at $\rho=R(y)$ and where

$$
B(s, x, y, \varepsilon, \lambda)=b_{1}\left(x(\rho / s)^{1 / Q},(s / \rho)^{q_{1} / Q} y_{1}, \ldots,(s / \rho)^{q_{n} / Q} y_{n}, \varepsilon, \lambda\right) .
$$

It can be readily verified that $\tilde{z}=\tilde{\varphi}(x, y, \varepsilon, \lambda)$ is an invariant graph under the local normal form. To that end, observe that $x^{Q} \rho$ and $y_{i}^{Q} / \rho^{q_{i}}$ are first integrals. Furthermore, this graph contains the initial manifold $\tilde{\gamma}$.

The $C^{1}$-smoothness of the invariant manifold $\tilde{z}=\tilde{\varphi}(x, y, \varepsilon, \lambda)$ follows directly from a study of the involved exponential, just like in Lemma 6 .

Lemma 9. Introduce the shortcut $K=\kappa_{0}-\kappa_{1} q_{1}-\cdots-\kappa_{n} q_{n}$. Let $m(x, y)<0$. Given any $\rho_{0}<1$, and any integer $N$, there exists $\varepsilon_{N}>0$ such that

$$
\left.\exp \left(\frac{-1}{Q \varepsilon^{r}|m(x, y)| \rho^{K / Q}} \int_{\rho}^{1} \frac{B(s, x, y, \varepsilon, \lambda)}{s^{1-K / Q}} d s\right)\right|_{\rho=R(y)} \leq C_{N}(R(y) \varepsilon|m|)^{N},
$$

for some $C_{N}>0$, as long as $0<\varepsilon \leq \varepsilon_{N}, x \in[-1,1]$, and $y$ with $0<R(y) \leq \rho_{0}$ and $m(x, y)<0$. When $m \not \equiv-1$, we may choose $\varepsilon_{N}=1$.

Proof. The proof is completely analogous to the proof of Lemma 6 .

The estimates in Lemma 9 imply that $z=\tilde{\varphi}(x, y, \varepsilon, \lambda)$ has a $C^{1}$-extension towards $\rho=0$ (i.e. towards $y=0$ ), and also towards $x=0$ and $\varepsilon=0$. Furthermore, the smoothness is only limited by the smoothness of the normal form and of the chosen initial manifold $\tilde{\gamma}$.

In original coordinates, this implies the existence of a $C^{1}$ center manifold $z=\varphi(x, y, \varepsilon, \lambda)$ containing the initial set $\gamma$. By techniques identical to those in Lemma 7 and Lemma 8, one can prove that this center manifold is in fact $C^{\infty}$ for $\varepsilon$ small enough, in case $m \not \equiv-1$. When $m \equiv-1$, we obtain $C^{k}$-smoothness for any $k$, provided we restrict $\varepsilon$ to a $k$-dependent neighborhood of 0 . This ends the proof of Theorem 1. 


\section{Proof of Theorem 2}

We look for a (sufficiently smooth) change of variables in the form

$$
z=u(1+\psi(x, y, u, \varepsilon, \lambda))
$$

with $\psi(x, y, 0, \varepsilon, \lambda)=0$, and impose that $\dot{u}=-a(x, y, \varepsilon, \lambda) u$ to find

$$
\begin{aligned}
u(1+\psi)^{2} F(x, y & , u(1+\psi), \varepsilon, \lambda) \\
= & \varepsilon^{r} m(x, y)\left(-x \psi_{x}+q_{1} y_{1} \psi_{y_{1}}+\cdots+q_{n} y_{n} \psi_{y_{n}}\right)-a u \psi_{u} .
\end{aligned}
$$

In other words, we search an invariant manifold of the form $v=\psi(x, y, u, \varepsilon, \lambda)$ for the family of vector fields

$$
\left\{\begin{aligned}
\dot{x} & =-x \varepsilon^{r} m(x, y), \\
\dot{y}_{i} & =q_{i} y_{i} \varepsilon^{r} m(x, y), \quad i=1, \ldots, n \\
\dot{u} & =-a u \\
\dot{v} & =u(1+v)^{2} F(x, y, u(1+v), \varepsilon, \lambda) .
\end{aligned}\right.
$$

This observation reduces the study of a near-identity normal form transformation to the study of an invariant manifold, and allows us to use techniques that are completely analogous to the techniques used in the construction of a center manifold for (1).

Like in Section 3, we first construct a solution of $(27)$ in $\{m(x, y)>0\}$. At the end of this section, we give a comment on how the set $\{m(x, y)<0\}$ is treated, and how both solutions can be extended smoothly to $m(x, y)=0$.

Using Theorem 3, applied to (3), we know the existence of a $C^{2}$ solution $\psi_{1}$ of $(27)$, locally, with the property $\psi_{1}(x, y, 0, \varepsilon, \lambda)=0$. Without loss of generality we may assume it is defined for $x, y_{i}, z \in[-1,1], \varepsilon \in[0,1]$.

Define the integers

$$
q^{i}=\frac{q_{1} q_{2} \ldots q_{n}}{q_{i}}
$$

We define the cylinder

$$
C: y_{1}^{2 q^{1}}+\cdots+y_{n}^{2 q^{n}}=1
$$

We now intend to adapt $\psi_{1}$ so that besides (27), also the boundary condition

$$
\left.\psi\right|_{C}=0
$$

is satisfied. For the moment we already know that $\left.\psi\right|_{C \cap\{u=0\}} \equiv 0$. To obtain (29), we exploit the non-uniqueness of solutions of (27), and observe that additional solutions can be found by composing the diffeomorphism $z=u(1+\psi)$ by another diffeomorphism that preserves the normal form. Let us write such a diffeomorphism, or better its inverse, in the form

$$
w=u(1+\beta(x, y, u, \varepsilon, \lambda))
$$

Imposing the boundary condition on the composition yields a condition on $\beta$ :

$$
\left.\beta\right|_{C}=\left.\psi_{1}\right|_{C}
$$


Imposing the invariance of the normal form under the transformation yields the following partial differential equation on $\beta$ :

$$
\varepsilon^{r} m(x, y)\left(-x \beta_{x}+q_{1} y_{1} \beta_{y_{1}}+\cdots+q_{n} y_{n} \beta_{y_{n}}\right)-a u \beta_{u}=0 .
$$

Remarkably, $\beta$ satisfies a linear partial differential equation, which we can solve using the method of characteristics:

Lemma 10. Equation (31) together with the boundary condition (30) has a unique $C^{2}$ solution on $\{m(x, y) \geq 0\}$, that is zero on $\{m(x, y)=0\}$.

Proof. With the method of characteristics in mind, we introduce the system of ordinary differential equations

$$
\left\{\begin{aligned}
\dot{x} & =-x \varepsilon^{r} m(x, y) \\
\dot{y}_{i} & =q_{i} y_{i} \varepsilon^{r} m(x, y), \\
\dot{u} & =-a(x, y, \varepsilon, \lambda) u \\
\dot{\beta} & =0
\end{aligned}\right.
$$

We introduce the radial variable

$$
\rho=R(y):=\sqrt{y_{1}^{2 q^{1}}+\cdots+y_{n}^{2 q^{n}}} .
$$

It can be readily verified that

$$
\dot{\rho}=Q \rho \varepsilon^{r} m(x, y) ; \quad \text { with } Q:=n . q_{1} \ldots q_{n},
$$

and that $x^{Q} \rho$ and $y_{i}^{Q} / \rho^{q_{i}}$ are constant along characteristic curves. The general solution of (31) is given by

$$
\begin{aligned}
& \beta(x, y, u, \varepsilon, \lambda)=\mathcal{B}\left(x \rho^{1 / Q}, \frac{y_{1}}{\rho^{q_{1} / Q}}, \ldots, \frac{y_{n}}{\rho^{q_{n} / Q}},\right. \\
&\left.u \exp \left(-\frac{1}{Q \varepsilon^{r} m(x, y) \rho^{K / Q}} \int_{\rho}^{1} \frac{A(s, x, y, \varepsilon, \lambda)}{s^{1-K / Q}} d s\right), \varepsilon, \lambda\right)\left.\right|_{\rho=R(y)},
\end{aligned}
$$

for any function $\mathcal{B}$, where $K=\kappa_{0}-q_{1} \kappa_{1}-\cdots-q_{n} \kappa_{n}$ and

$$
A(s, x, y, \varepsilon, \lambda)=a\left(x(\rho / s)^{1 / Q},(s / \rho)^{q_{1} / Q} y_{1}, \ldots,(s / \rho)^{q_{n} / Q} y_{n}, \varepsilon, \lambda\right) .
$$

(The appearing exponential coincides with the exponential in Lemma 9.) Evaluating $\beta$ at $C$, i.e. at $\rho=1$, we find $\beta(x, y, u, \varepsilon, \lambda)=\mathcal{B}(x, y, u, \varepsilon, \lambda)$, so the boundary condition is satisfied if we choose $\mathcal{B}=\psi_{1}$. We show the $C^{2}$-smoothness of $\beta$ for values of $y$ where $R(y)<1$. (Along $\{\rho=1, \varepsilon m(x, y)=0\}$ one cannot expect $\beta$ to be $C^{2}$; in general one finds a so-called boundary layer there.) In Lemma 9 it is shown that the exponential appearing inside (32) is flat w.r.t. $y$ at $y=0$ (or equivalently flat w.r.t. $\rho$ at $\rho=0$, since $\rho=R(y)$ ). This shows that

$$
\beta(x, y, u, \varepsilon, \lambda) \rightarrow \psi_{1}(0, *, 0, \varepsilon, \lambda), \quad \text { as } y \rightarrow 0,
$$

where $*$ can be any value $\tilde{y}$ with $R(\tilde{y})=1$. The continuity at $y=0$ follows from the fact that $\left.\psi_{1}\right|_{u=0}=0$. Similar arguments can be applied to show the continuity of any of the partial derivatives of $\beta$. Similarly, the exponential factor is flat w.r.t. $\varepsilon$ at $\varepsilon m=0$, from which one can derive the $C^{2}$-smoothness of $\beta$ at $\varepsilon m=0$. We remark that $C^{k}$-smoothness would follow as easily if the initial condition on $C$ (expressed by $\psi_{1}$ ) would have been $C^{k}$. 
Using Lemma 10, we find a solution of (27) subject to the boundary condition $\left.\psi\right|_{C}=0$, and which is $C^{2}$ for $\{\rho<1\} \cup\{\rho \leq 1, \varepsilon m \neq 0\}$. It is immediately clear from the method of characteristics that along regular characteristic curves, the solution is $C^{\infty}$ (or $C^{k}$ when $\varepsilon \leq \varepsilon_{k}$, depending on the smoothness of the initial system (3)). Characteristic curves through $(x, y, u)$ become singular for $\varepsilon=0$, for $m(x, y)=0$ or for $y=0$. In order to obtain better smoothness properties, we hence have to study these boundaries.

Using the method of characteristics, and using a covering of characteristic curves by neighborhoods in which local normal forms can be taken, we will now prove that such a solution is in fact $C^{\infty}$ on $\rho<1$ (respectively $C^{k}$ on $\rho<1$ when $\varepsilon \leq \varepsilon_{k}$ ). The method of proof is similar to the method used to deal with the smoothness of the center manifold in Theorem 1: we will cover a given characteristic curve through $\left(x_{*}, y_{*}, u_{*}\right)$ by neighborhoods in which we have local $C^{k}$-normal forms. Because the characteristic curves have a part near $u=0$ and a part away from $u=0$, we will need normal forms besides the normal forms of Lemma 4: away from $u=0$, we need a variant to the flow-box theorem, which partially puts a vector field in a flow box, leaving some directions fixed:

Lemma 11. Consider a $C^{k}$ vector field

$$
X_{\lambda}(v, z)=F(v, \lambda) \frac{\partial}{\partial v}+G(v, z, \lambda) \frac{\partial}{\partial z}
$$

defined for $v$ near 0 in $\mathbf{R}^{m}$, for $z$ near 0 in $\mathbf{R}$, and for $\lambda \in M$ ( $M$ is a compact in a finitely dimensional euclidean space). Assume that $F(0, \lambda)=0$ and $G(0,0, \lambda) \neq 0$. There exists an open neighborhood $V_{k}$ of the origin in $\mathbf{R}^{m+1}$ and a local $C^{k}$ family of diffeomorphisms of the form

$$
(v, z) \mapsto(v, \tilde{z}), \tilde{z}=\psi(v, z, \lambda),
$$

conjugating the family $X_{\mu}$ to the family

$$
F(v, \lambda) \frac{\partial}{\partial v}+1 \cdot \frac{\partial}{\partial \tilde{z}}
$$

Proof. This is an adapted version of the flow-box theorem. It suffices to use $(x, t)$ as variables instead of $(x, z)$, where $t$ is the time.

Lemma 12. The unique solution of (27) with boundary condition $\left.\psi\right|_{C}=0$ is smooth for $x .\|y\| \neq 0, m(x, y) \geq 0$. When (3) is $C^{\infty}$ along $x=0$, then so will $\psi$ be $C^{\infty}$ along $x=0$. Otherwise, $\psi$, defined on $m(x, y) \geq 0$, will be $C^{k}$ along $x=0$, provided $\varepsilon \leq \varepsilon_{k}$, for some sequence $\left(\varepsilon_{k}\right)_{k}$.

Proof. In this context we keep $y \neq 0$, so we only have to deal with the smoothness at $\varepsilon m=0$ (otherwise the value of $\psi$ is determined using an integration along regular characteristic curves). For any fixed $\left(x_{*}, y_{*}, u_{*}, \varepsilon_{*}\right)$ with $\varepsilon_{*} m\left(x_{*}, y_{*}\right)=0$, and for any $k$, we will show that $\psi$ is $C^{k}$ near $(x, y, u, \varepsilon)=\left(x_{*}, y_{*}, u_{*}, \varepsilon_{*}\right)$. Since $k$ is arbitrary, this will show the $C^{\infty}$-smoothness at $\varepsilon m=0$.

Let us look at a characteristic curve through $\left(x_{*}, y_{*}, u_{*}\right)$, as $\varepsilon \rightarrow 0$. It is an orbit of

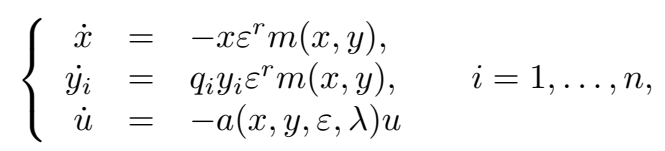



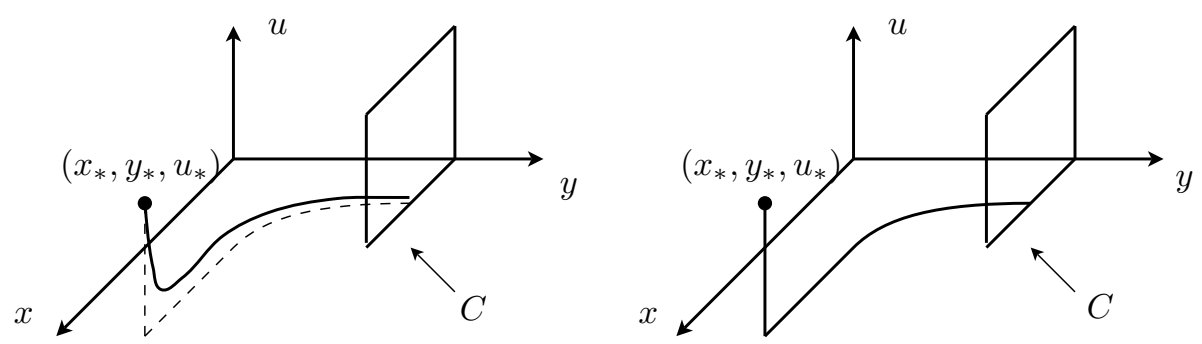

Figure 2: The characteristic curve through $\left(x_{*}, y_{*}, u_{*}\right)$ (when $\left.y_{*} \neq 0\right)$ to the left for $\varepsilon>0$, and the Hausdorff limit curve as $\varepsilon \rightarrow 0$ to the right.

and we consider the piece of the orbit from $\left(x_{*}, y_{*}, u_{*}\right)$ until the intersection point with $C$, see Figure 2. As $\varepsilon \rightarrow 0$, the characteristic curve tends towards a singular curve, see Figure 2. Similarly, when $m\left(x_{*}, y_{*}\right)=0$, characteristic curves through nearby $(x, y, u)$ tend towards a singular curve through $\left(x_{*}, y_{*}, u_{*}\right)$. We cover the singular curve by neighborhoods in which $C^{k}$-normal forms are valid, as in Lemma 4. Of course, this is only possible in the part of the curve where $u=0$. The part where $u \neq 0$ will be covered by neighborhoods where the "flowbox" normal form of Lemma 11 can be taken. Like in the proof of Lemma 7 (in order to deal with the smoothness of center manifolds), we choose points $\left(p_{1}, \ldots, p_{N}\right)$ on this limit curve, with $p_{N}=\left(x_{*}, y_{*}, u_{*}\right)$, with $p_{1}$ the intersection point of the limit curve with the plane $C$, in a way that the passage between $p_{i}$ and $p_{i+1}$ can be treated with one single local normal form. We then show by induction on $i$ that $\psi$ is smooth near $p_{i}$. Let us treat the induction step. Let

$$
z=u\left(1+\psi_{k}(x, y, u, \varepsilon, \lambda)\right)
$$

express a local $C^{k}$-normalizing transformation. The composition of $\psi$ with the inverse of the local transformation is expressed by

$$
z=u(1+\beta(x, y, u, \varepsilon, \lambda))
$$

where $\beta$ is a solution to (31). Furthermore, in the induction we may assume that $\beta$ is $C^{k}$ near $p_{i}$, and we have to prove that it is smooth near $p_{i+1}$. We discuss two cases: in the first case, $p_{i}$ and $p_{i+1}$ lay on $\{u=0\}$. In the second case, $p_{i}$ and $p_{i+1}$ lay both on the vertical part of the limit curve (see Figure 2). In the first case, we consider a manifold

$$
\left\{\rho=\rho_{i}, \beta=\beta_{i}(x, y, u, \varepsilon, \lambda)\right\}
$$

and consider characteristics through this manifold. We show that we can integrate $C^{k}$-smoothly to $\rho=\rho_{i+1}$, with $0<\rho_{i+1}<\rho_{i}$. This involves studying an expression like (32), as $\varepsilon m \rightarrow 0$, and is analogous to the discussion of the smoothness of $\beta$ in the proof of Lemma 10. In the second case, where $p_{i}$ and $p_{i+1}$ lay both on the vertical part of the limit curve, we can proceed as follows.

Even though at this stage, we cannot assume that the normalizing transformation expressed by $\varphi$ is $C^{k}$ near $p_{i+1}$, the resulting normal form is $C^{k}$ everywhere (because of its simple quasi-linear form). Therefore, we can apply the $C^{k}$-version of the flow-box lemma (Lemma 11) both to the original family of vector fields and to the family of vector fields after applying the normalizing 


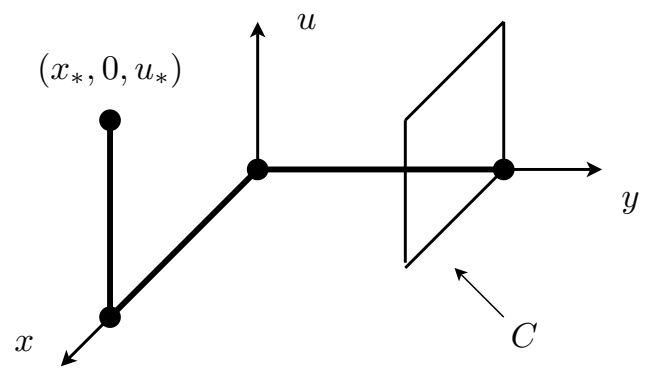

Figure 3: The characteristic curve through $\left(x_{*}, y_{*}, u_{*}\right)$, as $y_{*} \rightarrow 0$ (and $\varepsilon \rightarrow 0$ )

transformation expressed by $\varphi$. We now have two ways of locally getting to the flow-box form

$$
\left\{\begin{aligned}
\dot{x} & =-x \varepsilon^{r} m(x, y), \\
\dot{y} & =q_{i} y_{i} \varepsilon^{r} m(x, y), \quad i=1, \ldots, n, \\
\dot{u} & =1
\end{aligned}\right.
$$

One way directly with a $C^{k}$ flow-box transformation, another way by a composition of a flow-box transformation with the transformation expressed by $\psi$. By combining all these transformations, one gets a $C^{k}$-transformation

$$
z=u(1+\tilde{\beta}(x, y, u, \varepsilon, \lambda))
$$

conjugating the flow-box normal form (33) to itself, and for which we know that $\tilde{\beta}$ is $C^{k}$ near $p_{i}$. The $C^{k}$-smoothness of $\psi$ near $p_{i+1}$ is then a direct consequence of the $C^{k}$-smoothness of $\tilde{\beta}$ near $p_{i+1}$. Such a $\tilde{\beta}$ is a solution of the PDE

$$
\varepsilon^{r} m(x, y)\left(-x \tilde{\beta}_{x}+q_{1} y_{1} \tilde{\beta}_{y_{1}}+\cdots+q_{n} y_{n} \tilde{\beta}_{y_{n}}\right)-\tilde{\beta}_{u}=0,
$$

and we choose to integrate characteristic curves from the section $\left\{u=u_{i}\right\}$ near $p_{i}$, up to a section $\left\{u=u_{i+1}\right\}$. The equation is a regular perturbation of $\widetilde{\beta}_{u}=0$, and hence can be easily treated near $\varepsilon m=0$.

Lemma 13. Let $\psi$ be the unique solution of (27) with boundary condition $\left.\psi\right|_{C}=$ 0 , defined on $m(x, y) \geq 0$. Given $k \geq 1$, the function $\psi(x, y, \varepsilon, \lambda)$ is $C^{k}$ along $y=0$, for $\varepsilon$ sufficiently small. When $m \neq \equiv 1$ and when (3) is $C^{\infty}$, the function $\psi(x, y, \varepsilon, \lambda)$ is $C^{\infty}$ along $y=0$, for $\varepsilon$ sufficiently small.

Proof. We only have to treat the smoothness at $y=0$, and consider the Hausdorff limit of a characteristic curve through $\left(x_{*}, y_{*}, z_{*}\right)$ as $y_{*} \rightarrow 0$. This is shown in Figure 3. We just give a sketch of the proof, since the main ideas are identical to the ideas in Lemma 7, Lemma 8 and Lemma 12. We consider a covering of the limit curve drawn in Figure 3, and take a number of points $p_{1}, \ldots, p_{K}, \ldots, p_{L}, \ldots, p_{N}$, where $p_{1}$ is the point in $C, p_{N}=\left(x_{*}, y_{*}, u_{*}\right)$, and where $p_{K}$ and $p_{L}$ are the two intermediary corner points of the limit curve. The smoothness of $\psi$ near $p_{1}, \ldots, p_{K-1}$ follows from Lemma 12. The smoothness near $p_{K}$ follows the same way, if we notice that the expression for $\beta$ in (32) is sufficiently flat in $\rho$ as $\rho \rightarrow 0$ (and this way the transformation is $C^{k}$ at $\rho=0$ ).

The smoothness near $p_{K+1}, \ldots, p_{L}$ can be studied using a variant of the technique in Lemma 12: instead of integrating from section $\rho=\rho_{i}$ to $\rho=\rho_{i+1}$, 
we here need to integrate from sections $x=x_{i}$ to $x=x_{i+1}$. This yields a slightly different formula for $\beta$, but the study of the smoothness of $\beta$ is identical.

Finally, the part from $p_{L}$ to $p_{N}$ (the vertical part) is studied in a way identical to the study of the vertical part in the proof of Lemma 12.

Let us now comment on how the region $\{m(x, y) \leq 0\}$ is dealt with. Instead of imposing a boundary condition on $C$, we will now impose a boundary condition on $x= \pm 1$. The involved exponentials are exactly the same as those encountered in Section 3. We do not repeat the construction here, as it would be a repetition of the same ideas as before. The adaptation of the arguments presented above to the case $m \leq 0$ is hence left to the reader.

Now it only remains to be shown how the normalizing transformations in the different connected components of $m \neq 0$ can be seen as one smooth normalizing transformation. Again, we use the same ideas as in Section 3, but the proof is slightly different:

Lemma 14. Let $k$ be an arbitrary integer or $\infty$. Let $U_{1}, \ldots, U_{p}$ be the connected components of $[-1,1]^{n+1} \backslash\left\{m^{-1}(0)\right\}$. Suppose that $v=\psi(x, y, u, \varepsilon, \lambda)$ represents an invariant manifold of (28) and that $\psi(x, y, 0, \varepsilon, \lambda)=0$. When each $\psi_{i}=\left.\psi\right|_{\overline{U_{i}}}$ is $C^{k}$, then also $\psi$ is $C^{k}$.

Proof. The proof is analogous to the proof of Lemma 5. Suppose that $\kappa_{0} \neq 0$. Then some components $U_{\ell}$ and $U_{j}$ have a partly shared boundary on $\{x=0\}$. We show that $\psi$ is $C^{k}$ along $\{x=0\} \cap \overline{U_{\ell}} \cap \overline{U_{j}}$. Like in Lemma 5, the proof will follow from the uniqueness of formal power series expansion $z=\hat{\psi}:=$ $\sum_{i=0}^{\infty} \psi_{i}(y, u, \varepsilon, \lambda) x^{k}$ that express formally the invariance of $z=\hat{\psi}$. The zeroorder coefficient $\psi_{0}(y, u, \varepsilon, \lambda)$ in $\hat{\psi}$ is a solution of

$$
u\left(1+\psi_{0}\right)^{2} F(0, y, u(1+\psi), \varepsilon, \lambda)=-a u\left(\psi_{0}\right)_{u},\left.\quad\left(\psi_{0}\right)\right|_{u=0}=0 .
$$

It suffices to observe that this equation expresses the fact that the graph $v=$ $\psi_{0}(y, u, \varepsilon, \lambda)$ is the stable manifold of

$$
\left\{\begin{aligned}
\dot{u} & =-a u \\
\dot{v} & =u(1+v)^{2} F(0, y, u(1+\psi), \varepsilon, \lambda),
\end{aligned}\right.
$$

which is unique. Similarly, the higher order coefficients in this expansion can be seen as stable manifolds, and are hence all uniquely identified. Since both $\psi_{j}$ and $\psi_{\ell}$ are invariant manifolds of (28), their order $k$ Taylor expansion satisfies the above equation, and therefore their order $k$ Taylor expansion coincide.. This shows the $C^{k}$-smoothness along $\{x=0\} \cap \overline{U_{\ell}} \cap \overline{U_{j}}$. Similar arguments show the $C^{k}$-smoothness along $\left\{y_{i}=0\right\} \cap \overline{U_{\ell}} \cap \overline{U_{j}}$ when $\kappa_{i} \neq 0$.

This lemma, combined with the preceding results, finishes the proof of Theorem 2 .

\section{References}

[AM78] R. Abraham and J. E. Marsden, Foundations of mechanics, Benjamin/Cummings Publishing Co. Inc. Advanced Book Program, Reading, Mass., 1978, Second edition, revised and enlarged, With 
the assistance of Tudor Raţiu and Richard Cushman. MR 515141 (81e:58025)

[Bon96] P. Bonckaert, Partially hyperbolic fixed points with constraints, Trans. Amer. Math. Soc. 348 (1996), no. 3, 997-1011. MR 1321568 (96h:58157)

[Bon97] - Conjugacy of vector fields respecting additional properties, J. Dynam. Control Systems 3 (1997), no. 3, 419-432. MR 1472359 (99b:58214)

[DMD10] P. De Maesschalck and F. Dumortier, Singular perturbations and vanishing passage through a turning point, J. Differential Equations 248 (2010), no. 9, 2294-2328. MR 2595723

[DMD11] _ Detectable canard cycles with singular slow dynamics of any order at the turning point, Discr. Cont. Dyn. Syst. 29 (2011), no. 1, 109-140.

[DR] F. Dumortier and R. Roussarie, Smooth normal linearization of vector fields near lines of singularities, Qualitative Theory of Dynamical Systems, available online DOI: 10.1007/s12346-010-0020-y.

[DR96] , Canard cycles and center manifolds, Mem. Amer. Math. Soc. 121 (1996), no. 577, $\mathrm{x}+100$, With an appendix by Cheng Zhi Li. MR 1327208 (96k:34113)

[Tak71] F. Takens, Partially hyperbolic fixed points, Topology 10 (1971), 133147. MR 0307279 (46 \#6399)

[Tak86]_, A note on the differentiability of centre manifolds, Dynamical systems and partial differential equations (Caracas, 1984), Univ. Simon Bolivar, Caracas, 1986, pp. 101-104. MR 882016 (88f:58089)

[vS79] S. J. van Strien, Center manifolds are not $C^{\infty}$, Math. Z. 166 (1979), no. 2, 143-145. MR 525618 (80j:58049) 